\title{
Unified model of electron beam shot noise and coherent spontaneous emission in the helical wiggler free electron laser
}

\author{
B.W. J. McNeil, ${ }^{1}$ M.W. Poole, ${ }^{2}$ and G. R. M. Robb ${ }^{1}$ \\ ${ }^{1}$ Department of Physics, University of Strathclyde, Glasgow, G4 ONG, Scotland \\ ${ }^{2}$ ASTeC, Daresbury Laboratory, Warrington WA4 4AD, United Kingdom
}

(Received 23 December 2002; published 24 July 2003)

\begin{abstract}
A numerical algorithm for simulating electron beam shot noise in free electron lasers (FELs) is presented. Shot noise is a source of spontaneous emission that may be amplified in the self-amplified spontaneous emission regime of operation. This regime is of great importance to XUV and x-ray FELs where the spontaneous emission is currently the only effective source available for amplification. The algorithm uses a quasiuniform phase-space distribution of appropriately charge weighted macroparticles. The statistical properties of the macroparticles are derived directly from the temporal Poisson statistical properties of the real electron distribution. Unlike previous algorithms, ours does not rely upon any averaging over a resonant radiation period time scale and so more correctly describes the underlying physics. The algorithm also allows shot noise to be modeled self-consistently in unaveraged FEL models which are able to describe subwavelength phenomena such as coherent spontaneous emission (CSE). The algorithm is used in the unaveraged 1D FEL numerical simulation code FEMFEL and demonstrates spontaneous emission due to shot noise and CSE in both rectangular and Gaussian electron pulse current profiles. The preliminary results show good qualitative and quantitative agreement with theory.
\end{abstract}

DOI: 10.1103/PhysRevSTAB.6.070701

PACS numbers: 52.59.Rz, 41.60.Cr, 61.80.Fe

\section{INTRODUCTION}

The free electron laser (FEL) is an important source of high power tunable radiation that spans a wide range of the electromagnetic spectrum from the microwave to VUV and potentially beyond. In the XUV and $\mathrm{x}$-ray regions there are currently few or no effective sources available for amplification by an FEL amplifier and the FEL must start up from noise, operating in the selfamplified spontaneous emission (SASE) regime [1]. The source of spontaneous radiation is the electron beam "shot noise" arising from the random distribution of electron arrival times at the beginning of the FEL interaction region. In the SASE regime this radiation is exponentially amplified as it copropagates with the electron beam through the FEL interaction region. Any computer code that attempts to model the SASE FEL must therefore have a valid numerical model of the electron beam shot noise to be able to simulate the radiation amplification process.

Current computer memory and CPU limitations make it impractical for FEL computer simulation codes to follow the evolution of each individual electron in a beam. The electron distribution is therefore replaced by a distribution of "macroparticles" designed to simulate the real electron distribution. In order to model shot noise, the macroparticle distribution should therefore simulate the statistical properties of the real electron distribution. Many, if not most simulation codes such as GINGER [2] and GENESIS 1.3 [3] currently use the algorithm of [4] as the basis of their macroparticle loading algorithm to describe shot noise. The algorithm has been adapted and extended in ingenious ways to include higher dimensions of electron phase space and harmonics of the radiation field (see, e.g., [5] for a recent discussion).

However, the algorithm of [4], although effective, has been derived directly from the statistical properties of an averaged quantity, $b$, the bunching parameter [1]. This averaging occurs over a resonant radiation period. There has not been, to the authors' knowledge, a contiguous derivation from the statistical properties of the individual electrons in an electron beam to the algorithm of [4]. Given the large resources that are being devoted to the design and construction of new short wavelength SASE FELs such as that at the TESLA facility in Germany [6], the proposed LCLS facility at SLAC [7] in the U.S., and the U.K. 4GLS project [8], it would seem timely and prudent to derive a particle loading algorithm from first principles that may be traced directly back to the physics of the individual electrons.

Such a model would also allow the introduction of shot noise into FEL models that have not been averaged over a radiation period in a consistent way. Although the method of [4] has been used in unaveraged models such as [9] it cannot be considered consistent to use methods developed from averaged equations in an unaveraged model.

Some previous work was carried out to model distributions of electrons which begins with the assumption that electron arrival at the beginning of the interaction region of a FEL is a Poisson process [10]. However, this work was concerned with deriving averaged quantities of the bunching and did not attempt to describe a macroparticle model. The work presented here derives such a 
macroparticle model directly from a Poisson statistical electron distribution and demonstrates its use in a one dimensional finite element code FEMFEL currently under development.

\section{THE SHOT-NOISE MODEL}

The arrival of electrons at the beginning of an interaction region, $z=0$, is assumed to be a Poisson process. We first discretize time into small intervals of uniform duration $\Delta t$ so that $t_{n}=n \Delta t$, where $n=0, \pm 1, \pm 2, \ldots$. In the notation hereafter subscript $n$ always refers to these discrete times. It will be seen that the time interval $\Delta t$ is the mean interval between macroparticles introduced to model the real electron distribution. Furthermore, $\Delta t$ is small with respect to any radiation period to be subsequently modeled, i.e., $\Delta t \ll 2 \pi / \omega_{\max }$.

Consider the arrival of electrons over one such time interval $t_{n} \leq t<t_{n+1}$. The mean rate of the Poisson process is the rate of electron arrival $\nu_{n}=I\left(t_{n}\right) / e$ which is assumed constant over the interval $\Delta t$. The electron arrival times obey Poisson statistics and the number of electrons, $N_{n}$, arriving within the interval $\Delta t$ is a statistical variable determined by the Poisson distribution:

$$
P\left(N_{n}\right)=\frac{\bar{N}_{n}^{N_{n}} e^{-\bar{N}_{n}}}{N_{n} !}
$$

where $\bar{N}_{n}=\nu_{n} \Delta t$, is the expectation for the number of electrons in the interval $\Delta t$. It can be shown [11] that the ordered arrival times of the electrons have identical statistical properties to those with unordered arrival times each of which have been distributed within the interval $\Delta t$ with an identical uniform probability density $p_{n}=$ $\nu_{n} / \bar{N}_{n}=1 / \Delta t$. The statistics of variables distributed with uniform probability density over a finite interval are well known [12] from which we obtain the mean and variance of each of the unordered electron arrival times $t_{j}, j=1, \ldots, N_{n}$ to be

$$
\mu_{n}=t_{n}+\Delta t / 2, \quad \sigma_{n}^{2}=\Delta t^{2} / 12,
$$

respectively.

For a total of $N_{n}$ electrons the mean arrival time is given by

$$
\bar{t}_{n}=\frac{1}{N_{n}} \sum_{j=1}^{N_{n}} t_{j}
$$

with expectation and variance of $\bar{t}_{n}$ easily shown to be

$$
E\left(\bar{t}_{n}\right)=\bar{\mu}_{n}=\mu_{n}, \quad V\left(\bar{t}_{n}\right)=\bar{\sigma}_{n}^{2}=\frac{\sigma_{n}^{2}}{N_{n}}=\frac{\Delta t^{2}}{12 N_{n}},
$$

respectively.

The distribution of the $N_{n}$ electrons within the interval $t_{n} \leq \Delta t \leq t_{n+1}$ may now be modeled by replacing the distribution with a single macroparticle whose statistical properties of charge and temporal distribution equal those of the $N_{n}$ electrons. This is the physical basis of the model presented here.

The statistical properties of the macroparticle charge are given simply by the Poisson distribution (1). When loading the macroparticles in a numerical simulation code each would be assigned a charge weight of $Q_{n}=$ $N_{n} e$ where $e$ is the charge of an electron and $N_{n}$, the macroparticle electron number, would be generated by a Poisson random deviate generator of mean $\bar{N}_{n}$.

The statistical properties of the macroparticle arrival time may be found by first placing the macroparticle at the mean electron distribution arrival time $\bar{\mu}_{n}$. This mean arrival time then has added to it an independent random variable $\tau$ with uniform probability distribution over the interval $[-\delta t / 2, \delta t / 2]$. The interval $\delta t$ is chosen so that the variance in the macroparticle arrival time is equal to that of the real electron distribution (3). Similarly to relation (2) the variance for the macroparticle arrival time is $\delta t^{2} / 12$. Equating this macroparticle variance to that of the real electron distribution (3) the following relation for $\delta t$ is obtained:

$$
\delta t=\frac{\Delta t}{\sqrt{N_{n}}} \approx \frac{\Delta t}{{\sqrt{\bar{N}_{n}}}^{\prime}}
$$

where the latter approximation may be used when $\bar{N}_{n} \gg 1$.

Another method of determining the statistics of the macroparticle arrival time makes use of the central limit theorem [12]. In the limit $N_{n} \rightarrow \infty$ this theorem allows the real distribution of electrons to be replaced by a single macroparticle with an arrival time described by a random Gaussian deviate with mean $\bar{\mu}_{n}$ and standard deviation $\bar{\sigma}_{n}$. However, practical experience has shown that the uniform deviate method leading to (4) works equally well if not better than the central limit theorem method, and this is the method used hereafter.

An electron pulse defined by a mean current $I(z, t)$ may be modeled to include the effects of shot noise by assigning macroparticles, with charge and arrival time as described above, over many such consecutive time intervals defined by $t_{n}$. The macroparticle arrival times describing the complete electron pulse may then be written as

$$
t_{j}=\bar{t}_{j}+\tau_{j}, \quad j=1, \ldots, N_{m},
$$

where $N_{m}$ is the total number of macroparticles and the $j=1$ macroparticle arrives in the $n$th time interval so that $\bar{t}_{j}=t_{n}+(j-1 / 2) \Delta t$. For consistency, the Poisson variate $N_{n}$, the electron number for the $j$ th macroparticle, is now written as $N_{j}$.

It is important to note that the electron distribution and its statistics have been modeled by the macroparticles without reference to any external lengths or time scales such as a resonant radiation frequency or its harmonics. In this sense this analysis is self-consistent. 


\section{BUNCHING STATISTICS IN 1D}

The following scaled form of the $1 \mathrm{D}$ wave equation describing the FEL interaction may be derived:

$$
\left(\frac{\partial}{\partial \bar{z}}+\frac{\partial}{\partial \bar{z}_{1}}\right) A\left(\bar{z}, \bar{z}_{1}\right)=\frac{1}{\bar{n}_{p \|}} \sum_{j=1}^{N} \exp \left(-i \frac{\bar{z}_{1}}{2 \rho}\right) \delta\left(\bar{z}_{1}-\bar{z}_{1 j}\right) .
$$

The magnetic undulator field and the electric field of the fundamental radiation frequency used in the derivation of (6) are, respectively,

$$
\begin{gathered}
\mathbf{B}_{w}(z)=\frac{B_{w}}{\sqrt{2}}\left(\hat{\mathbf{e}} e^{-i k_{w} z}+\text { c.c. }\right), \\
\mathbf{E}(z, t)=-\frac{1}{\sqrt{2}}\left[\hat{\mathbf{e}} E(z, t) e^{i(k z-\omega t)}+\text { c.c. }\right],
\end{gathered}
$$

where $\hat{\mathbf{e}}=(\hat{\mathbf{x}}+i \hat{\mathbf{y}}) / \sqrt{2}$. The scaling of the field used in deriving $(6)$ is given by $[1,13]$

$$
\begin{gathered}
\mathbf{A}(z, t)=\frac{e \mathbf{E}(z, t)}{m c \omega_{p} \sqrt{\gamma \rho}}, \\
\bar{z}=2 k_{w} \rho z, \\
\bar{z}_{1}=\frac{2 k_{w} \rho}{1-\bar{\beta}_{z}}\left(z-c \bar{\beta}_{z} t\right) .
\end{gathered}
$$

It is important to note that no slowly varying approximations have been made regarding the scaled complex field envelope $A(z, t)$. All frequency components must be modeled by the envelope $A(z, t)$, which, when containing components that differ significantly from the fundamental frequency, may result in relatively rapid modulations of the envelope over a fundamental optical period. Analysis of specific frequencies, or frequency ranges, therefore requires a Fourier analysis of the field (9).

The right-hand side of the wave equation is written in terms of the real electron distribution, where $N$ is the total number of electrons in the electron pulse and at the beginning of the interaction region, $z=0$, so that $\bar{z}_{1}=$ $-2 \rho \omega t$. Note that the total number of electrons in the electron pulse, $N$, is itself a Poisson variate of mean $\bar{N}$, the expectation value of the total electron number given by

$$
\bar{N}=\int_{-\infty}^{\infty} \frac{I(t)}{e} d t
$$

The Dirac delta function transforms to real units as

$$
\delta\left(\bar{z}_{1}-\bar{z}_{1 j}\right)=\frac{\delta\left(\omega t-\omega t_{j}\right)}{2 \rho},
$$

and the electron distribution may be replaced by a macroparticle distribution as described in the previous section to give the right-hand side of the wave equation (6) in terms of the macroparticle distribution

$$
\frac{2 \pi}{N_{m \lambda}} \sum_{j=1}^{N_{m}} \frac{N_{j}}{\bar{N}_{\mathrm{pk}}} e^{i \omega t} \delta\left(\omega t-\omega t_{j}\right)
$$

where $N_{m \lambda}=2 \pi / \omega \Delta t$ is the number of macroparticles within one radiation period and $\bar{N}_{\mathrm{pk}}=I_{\mathrm{pk}} \Delta t / e$ is the expectation of the macroparticle electron number at the peak of the electron pulse current, $I_{\mathrm{pk}}$.

When averaged over a radiation period the right-hand side of the wave equation yields a quantity known as the "bunching parameter" [1]. If the macroparticle model is valid then the statistics of the bunching parameter must be the same for the macroparticle distribution as for a real electron distribution, and we now test for this.

Averaging (14) by integrating over one radiation period centered at time $t$ yields an expression for the localized macroparticle bunching parameter:

$$
b(t)=\frac{1}{N_{m \lambda}} \sum_{j=1}^{N_{m \lambda}} \frac{N_{j}}{\bar{N}_{\mathrm{pk}}} e^{i \omega \tau_{j}} e^{i \omega \bar{t}_{j}}
$$

The delta function has extracted those macroparticles within the interval (we retain $j$ as the index for simplicity) and we have used relation (5).

The expectation of the bunching parameter (15) is then given as

$$
E(b)=\frac{1}{N_{m \lambda}} \sum_{j=1}^{N_{m \lambda}} E_{N_{j}}\left(\frac{N_{j}}{\bar{N}_{\mathrm{pk}}} E_{N_{j} \mid \tau_{j}}\left(e^{i \omega \tau_{j}}\right)\right) e^{i \omega \bar{t}_{j}},
$$

where $E_{N_{j}}(\cdots)$ signifies the expectation value with respect to the macroparticle electron number $N_{j}$, and $E_{N_{j} \mid \tau_{j}}(\cdots)$ signifies the expectation value with respect to the randomness of the macroparticle arrival time $\tau_{j}$ for a given value $N_{j}$. The latter expectation is obtained by averaging in $\tau_{j}$ over the interval $[-\delta t / 2, \delta t / 2]$ from which, using relation (4), the following result is obtained:

$$
E_{\tau_{j} \mid N_{j}}\left(e^{i \omega \tau_{j}}\right)=\frac{\sqrt{N_{j}} N_{m \lambda}}{\pi} \sin \left(\frac{\pi}{\sqrt{N_{j}} N_{m \lambda}}\right) .
$$

Substituting for (17) into (16), using the result that for a Poisson distribution $E_{N_{j}}\left(N_{j}\right)=\bar{N}_{j}$, and assuming the usually easily satisfied condition $\sqrt{N_{j}} N_{m \lambda} \gg 1$ so that

$$
\sin \left(\frac{\pi}{\sqrt{N_{j}} N_{m \lambda}}\right) \approx \frac{\pi}{\sqrt{N_{j}} N_{m \lambda}},
$$

the expression for the expectation of the bunching is

$$
E(b)=\frac{1}{N_{m \lambda}} \sum_{j=1}^{N_{m \lambda}} \frac{\bar{N}_{j}}{\bar{N}_{\mathrm{pk}}} e^{i \omega \bar{t}_{j}} .
$$

A similar but more lengthy analysis for the expectation of $|b|^{2}$ may also be carried out to obtain 


$$
E\left(|b|^{2}\right)=\frac{1}{N_{m \lambda}^{2} \bar{N}_{\mathrm{pk}}^{2}} \sum_{j=1}^{N_{m \lambda}} \bar{N}_{j}+|E(b)|^{2}
$$

from which an expression for the variance of the bunching, $V(b)=E\left(|b|^{2}\right)-|E(b)|^{2}$, is obtained.

It can be seen from (18) for the expression for $E(b)$ that if the current is not uniform over the radiation period, i.e., $\bar{N}_{j} \neq$ const $\forall j$, then $E(b)$ will be nonzero. Such nonzero bunching is caused by a current gradient and is the source of coherent spontaneous emission (CSE) [13].

In the limit of a uniform current beam we may set $\bar{N}_{j}=$ $\bar{N}_{\mathrm{pk}} \forall j, E(b)=0$, and there is no CSE. In this case $\bar{N}_{m \lambda} \bar{N}_{\mathrm{pk}}=\bar{N}_{\lambda}$, the expectation of the total number of electrons in the radiation period, and we obtain from (19) the result that $E\left(|b|^{2}\right)=1 / \bar{N}_{\lambda}$, which for the single radiation period under consideration here, is in agreement with the previous analysis of averaged Poisson statistical models [10].

\section{HIGHER PHASE-SPACE DIMENSIONS}

In the above analysis, electron beam properties such as energy and transverse momentum spread were neglected. In order to describe these effects and include the effects of shot noise, the above model must be extended to a multidimensional electron phase space.

Phase space is first discretized into elemental "volumes" by discretizing along each phase-space ordinate in a method similar to that carried out for time in the previous section. The populating by electrons of each phase-space "volume element" at $z=0$ is then assumed to be a Poisson process with each element being populated at a local Poisson rate given by

$$
\nu(\boldsymbol{\alpha}, t)=\frac{I(t)}{e} f(\boldsymbol{\alpha}),
$$

where $\boldsymbol{\alpha}$ is a generalized phase-space coordinate [e.g., $\boldsymbol{\alpha}=\left(\mathbf{r}_{\perp}, \mathbf{p}\right)$, the transverse coordinate and the momentum, respectively] and $f(\boldsymbol{\alpha})$ is a normalized distribution function. Note that, in general, the distribution function itself may have a temporal dependence via $\boldsymbol{\alpha}$.

The same algorithm as was used for allocating the temporal noise of the previous section is now used for each phase-space coordinate of the macroparticles. The macroparticles are placed at the "center" of each phasespace volume element and have added to each of their phase-space coordinates an independent random variable of uniform probability distribution. This random variable is equivalent to the $\tau_{j}$ of the previous section and, for a generalized ordinate $\alpha_{k}$, will have a range

$$
\left[-\frac{\Delta \alpha_{k}}{2 \sqrt{\bar{N}}_{j}}, \frac{\Delta \alpha_{k}}{2{\sqrt{\bar{N}_{j}}}}\right]
$$

where $\Delta \alpha_{k}$ is the discretization interval and from (20)

$$
\bar{N}_{j}=\nu(\boldsymbol{\alpha}, t) \Delta V_{\alpha} \Delta t=\frac{I(t)}{e} f(\boldsymbol{\alpha}) \Delta V_{\alpha} \Delta t,
$$

where $\Delta V_{\alpha}=\prod_{k}\left(\Delta \alpha_{k}\right)$ is the elemental phase-space volume. Each macroparticle will also have assigned to it a Poisson random variate electron number of mean $\bar{N}_{j}$.

\section{Numerical example}

We now demonstrate the use of the macroparticle model above by considering the bunching in a uniform current electron beam of $1 \mathrm{kA}$ with a uniformly distributed relative energy spread of half width $\sigma_{\gamma} / \gamma=\rho / 2$ at a radiation wavelength of $10 \mathrm{~nm}$ and with a FEL parameter of $\rho=10^{-3}$. These parameters are typical of a Compton regime SASE FEL operating in the XUV. In the notation of [13] the energy spread above may be described as a spread in the scaled energy parameter $p$ as $\sigma_{p}=1 / 2$.

With these parameters then in one radiation period approximately $\bar{N}_{\lambda}=2 \times 10^{5}$ electrons are distributed with a uniform random distribution over phase space defined by the limits $0<\bar{z}_{1}<4 \pi \rho$ and $-\sigma_{p}<p<\sigma_{p}$. The normalized distribution function $f(p)=1 / 2 \sigma_{p}=1$ over this latter interval and is zero elsewhere. The area so defined is discretized uniformly in $\bar{z}_{1}$ and $p$ to form a $10 \times 10$ grid with $\Delta \bar{z}_{1}=4 \pi \rho / 10$ and $\Delta p=2 \sigma_{p} / 10$. Each macroparticle will have assigned to it a Poisson variate electron number of mean $\bar{N}_{j}=2 \times 10^{3} \quad \forall j$ and will be placed at the mean position (center) of each grid element about which it will have a uniform deviate of range defined by (21) added to this mean position. In $\bar{z}_{1}$ the range is over $\pm \pi \rho / 5 \sqrt{\bar{N}_{j}} \approx \pm 1.4 \times 10^{-5}$, and in $p$ the range is over $\pm \sigma_{p} / 10 \sqrt{\bar{N}_{j}} \approx \pm 1.1 \times 10^{-3}$.

The bunching statistics of an ensemble of $10^{5}$ such macroparticle distributions are now compared with those statistics expected from the real electron distribution of $2 \times 10^{5}$ electrons.

The bunching parameter $b$ as defined by (15) was calculated for each independent distribution of the ensemble and the bunching statistics calculated. The probability distribution of $|b|$ and $|b|^{2}$ of the ensemble was collated and plotted as histograms in Figs. 1 and 2, respectively. The probability distribution of $|b|$ for a real distribution of electrons has previously been shown to be the Rayleigh distribution, and that of $|b|^{2}$ the negative exponential distribution [14]. These distribution functions are plotted for comparison in Figs. 1 and 2 as solid lines and show that the probability distributions of the macroparticle bunching statistics are in good agreement with those of a real electron distribution.

The above probability distribution functions yield the following relations for an ensemble of real electron distributions [5]: $\quad b_{\mathrm{rms}}=\sqrt{\left\langle|b|^{2}\right\rangle} \approx 1 / \sqrt{\bar{N}_{\lambda}} ; \quad \bar{b}=\langle|b|\rangle \approx$ $\sqrt{\pi / 4 \bar{N}_{\lambda}}$, and $\sigma_{|b|}=\sqrt{\left\langle(b-\bar{b})^{2}\right\rangle} \approx \sqrt{1-\pi / 4} / \sqrt{\bar{N}_{\lambda}}$, where, as previously, $\bar{N}_{\lambda}$ is the expectation of the number 


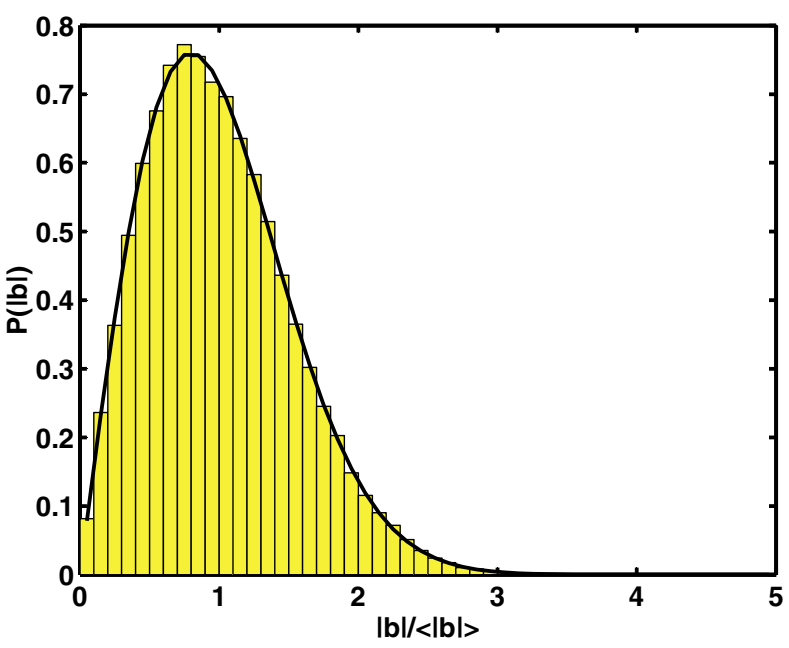

FIG. 1. (Color) The probability distribution $P(|b|)$ for the ensemble of $10^{5}$ macroparticle distributions as a histogram. The solid line is the Rayleigh distribution function of mean equal to 1 .

of electrons in the radiation period. These quantities were calculated numerically from the ensemble of macroparticle distributions and compared with the results above for the real electron distribution with $\bar{N}_{\lambda}=2 \times 10^{5}$ which are given in brackets: $b_{\mathrm{rms}}=2.27 \times 10^{-3}\left(2.24 \times 10^{-3}\right)$; $\bar{b}=2.01 \times 10^{-3} \quad\left(1.98 \times 10^{-3}\right) ; \quad \sigma_{|b|}=1.05 \times 10^{-3}$ $\left(1.04 \times 10^{-3}\right)$.

Therefore, it can be concluded that the macroparticle distribution gives a good approximation to the bunching statistics of the real electron distribution.

An investigation of the statistics of a macroparticle distribution has also been performed for the case of a drifting electron beam by loading the macroparticles as

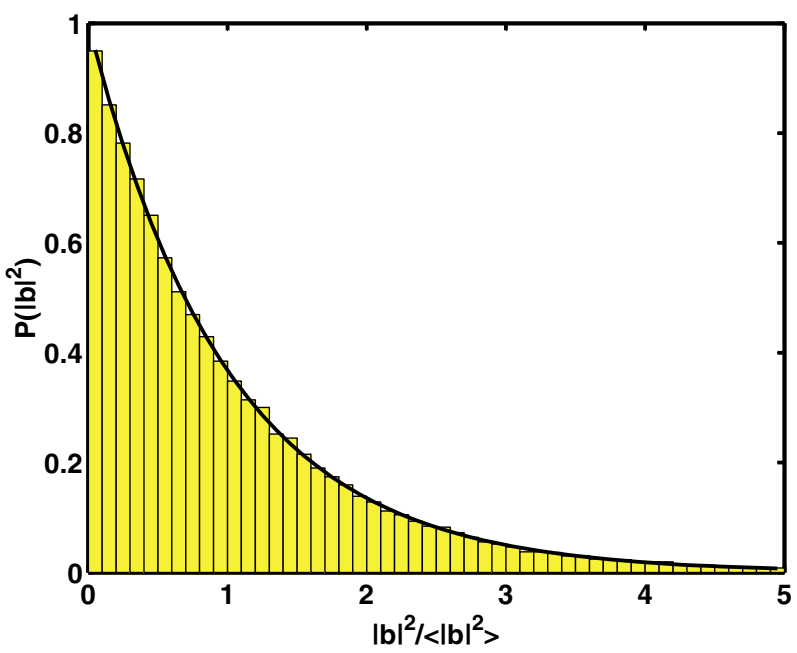

FIG. 2. (Color) The probability distribution $P\left(|b|^{2}\right)$ for the ensemble of $10^{5}$ macroparticle distributions as a histogram. The solid line is the negative exponential distribution function of mean equal to 1 . above and integrating the FEL equations [13] in the absence of any radiation field. Identical parameters as above were used to simulate shot noise in a section of a constant current electron beam and the beam was allowed to drift a scaled distance of $\bar{z}=1$. Because of the spread $\sigma_{p}$ in the scaled energy parameter $p$, the macroparticles also drift with respect to each other in phase $\bar{z}_{1}$. They also drift across the boundaries of the interval $\Delta \bar{z}_{1}=4 \pi \rho$ over which the bunching parameter $b$ is calculated, and as the beam is of constant current the average flow of macroparticles into this interval is equal to the average flow out of it. For such a region, no perceptible change in the bunching statistics, as demonstrated by Figs. 1 and 2 above, has been observed as a function of the drift position $\bar{z}$. This demonstrates that there are no correlations of the ensemble average bunching statistics with drift position.

\section{THE ALGORITHM IN USE}

The above noise algorithm is now applied in an unaveraged 1D FEL simulation code currently under development to describe the effects of shot noise, CSE, and energy spread. This code, which we call FEMFEL, has been developed from previous codes describing the effects of CSE $[9,13,15]$ by using the finite element method (FEM) to numerically solve the wave equation. The output data of this code are in SDDS format [16] and postprocessing and plotting has been carried out using the SDDS tool kit [17].

The code was used to model a Compton regime FEL over the first five gain lengths of the interaction region which in the variables used here [13] gives a scaled interaction length of $\bar{z}=5$. The fundamental FEL parameter $\rho=0.1 / 4 \pi \approx 7.96 \times 10^{-3}$ so that there are ten ponderomotive periods per unit $\bar{z}_{1}$. The electron pulse current is assumed to be a rectangular, or "top hat," function of total charge $Q=1 \mathrm{nC}$ and of scaled duration in $\bar{z}_{1}$ of $\bar{l}_{e}=6$. A Gaussian energy spread distribution is used with $\sigma_{p}=0.5$ over a full range $-3 \sigma_{p}<p<3 \sigma_{p}$. Electron phase space was discretized into a uniform $40 \times 21$ grid in $\left(\bar{z}_{1}, p\right)$ per ponderomotive period giving a total of 840 macroparticles per period and therefore a total of 50400 macroparticles in the 60 period pulse. The average macroparticle therefore represents $\approx 1.25 \times 10^{5}$ electrons.

For this rectangular profile pulse, the scaled linear density in the wave equation (6) may be written as $\bar{n}_{p \|}=$ $\bar{N} / \bar{l}_{e}$, where, as previously, $\bar{N}$ is the expectation of the total number of electrons in the pulse, allowing the wave equation to be written

$$
\left(\frac{\partial}{\partial \bar{z}}+\frac{\partial}{\partial \bar{z}_{1}}\right) A\left(\bar{z}, \bar{z}_{1}\right)=\bar{l}_{e} \sum_{j=1}^{N_{m}} \frac{N_{j}}{\bar{N}} \exp \left(-i \frac{\bar{z}_{1}}{2 \rho}\right) \delta\left(\bar{z}_{1}-\bar{z}_{1 j}\right) .
$$


The streamline method of finite element analysis [18] was used to model the evolution of the radiation pulse in $\bar{z}_{1}$, with iteration forward in $\bar{z}$ being governed by a CrankNicolson scheme [19].

The macroparticles are assigned to the phase-space grid as described in the previous section. This is shown schematically in Fig. 3.

Each macroparticle has assigned to it a Poisson random variate $N_{j}$ of mean $\bar{N}_{j}$ obtained from Eq. (22) which for the rectangular profile pulse used here is given by

$$
\bar{N}_{j}=\frac{\bar{N}}{\bar{l}_{e}} \frac{1}{\sqrt{2 \pi} \sigma_{p}} \exp \left(-\frac{p_{g j}^{2}}{2 \sigma_{p}^{2}}\right) \Delta p \Delta \bar{z}_{1},
$$

where $p_{g j}$ refers to the mean grid position of the $j$ th macroparticle. This randomness in the number of electrons each macroparticle represents is demonstrated schematically by the differing sizes of the macroparticles in Fig. 3.

The macroparticles are placed at the center of each grid cell $\left(\bar{z}_{1 g j}, p_{g j}\right)$ and then have a uniform random variate determined by Eq. (21) added to this mean coordinate, i.e.,

$$
\left(\bar{z}_{1 j}, p_{j}\right)=\left(\bar{z}_{1 g j}, p_{g j}\right)+\left(U_{z_{1}}, U_{p}\right)
$$

where $U_{z_{1}}$ and $U_{p}$ are uniform random variates over the intervals

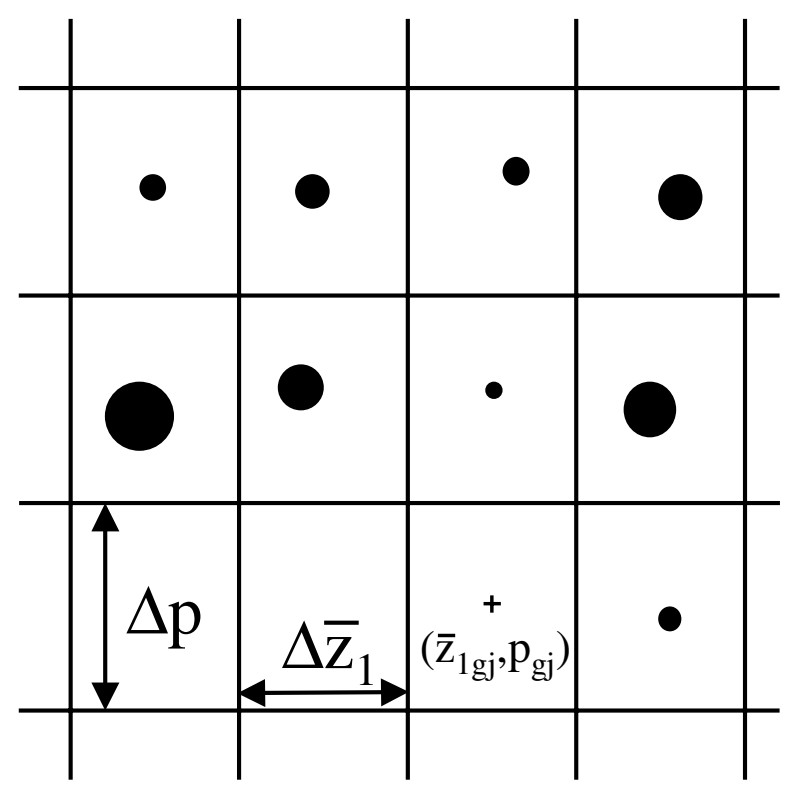

FIG. 3. Schematic of macroparticle loading algorithm. The bottom left three grid boxes show the notation used for the grid dimensions and center. The randomness in the macroparticle charge via the Poisson random variate $N_{j}$ is demonstrated via their different relative sizes. The noise due to the random positioning is demonstrated via the variation about the grid centers.

$$
\left[-\frac{\Delta \bar{z}_{1}}{2{\sqrt{\bar{N}_{j}}}_{j}}, \frac{\Delta \bar{z}_{1}}{2{\sqrt{\bar{N}_{j}}}}\right]
$$

and

$$
\left[-\frac{\Delta p}{2 \sqrt{\bar{N}}_{j}}, \frac{\Delta p}{2 \sqrt{\bar{N}_{j}}}\right],
$$

respectively. The randomness in the macroparticle phasespace distribution is demonstrated schematically in Fig. 3 by the random deviation of the particle positions from the grid centers.

The equations of motion for the $j=1, \ldots, N_{m}$ macroparticles in the radiation field are those of Ref. [13]:

$$
\begin{gathered}
\frac{d \bar{z}_{1 j}}{d \bar{z}}=2 \rho p_{j}, \\
\frac{d p_{j}}{d \bar{z}}=-\left[A\left(\bar{z}, \bar{z}_{1 j}\right) \exp \left(\frac{\bar{z}_{1 j}}{2 \rho}\right)+\text { c.c. }\right] .
\end{gathered}
$$

Figure 4 shows the scaled "intensity" $|A|^{2}$ and power spectral density $P$ that results from a run of the code FEMFEL for the above parameters and macroparticle loading algorithm, and plotted using the SDDS tool kit package [17]. Strictly, $|A|^{2}$, as defined here, is the scaled magnitude of the $z$ component of the Poynting vector, whereas intensity is normally defined as the timeaveraged magnitude of the Poynting vector. For brevity, we use intensity here in the former, unaveraged, sense. The scaled power spectral density, $P$, is the summation of the PSDs of the scaled field components $A_{x}$ and $A_{y}$, as obtained from the field definitions (8) and (9). The frequency, $f$, is scaled with respect to the fundamental
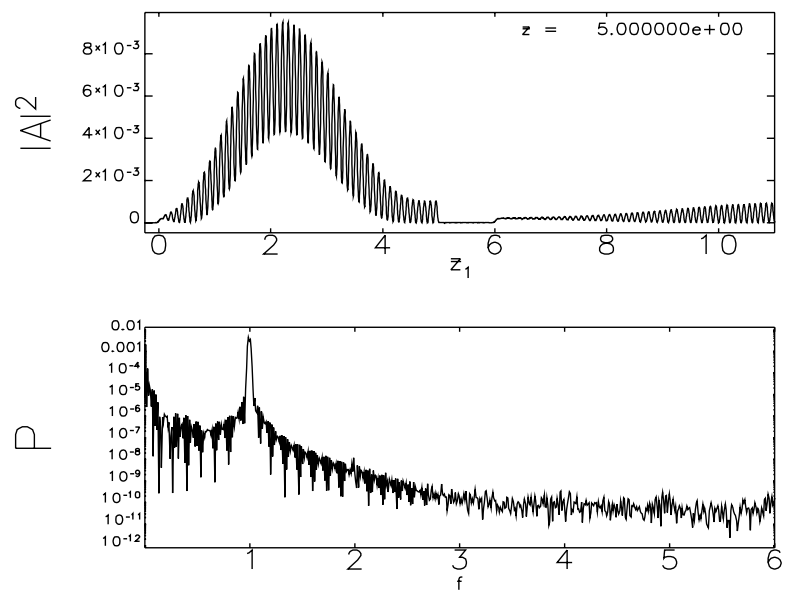

FIG. 4. Top panel: The scaled intensity, $|A|^{2}$, as a function of scaled pulse position $\bar{z}_{1}$ for a scaled distance through the interaction region of $\bar{z}=5$ and a rectangular profile electron pulse duration of $\bar{l}_{e}=6$ between $0<\bar{z}_{1}<6$. The slippage region is $0<\bar{z}_{1}<5$; the steady-state region is $5<\bar{z}_{1}<6$; the vacuum region is $\bar{z}_{1}>6$. Bottom panel: The scaled PSD, $P$, of the radiation as a function of the scaled frequency $f$. 
resonant FEL frequency. These PSDs were generated by the SDDS tool kit function SDDSFFT [17].

The dominant features of the radiation evolution are essentially those of self-amplified coherent spontaneous emission (SACSE) in the presence of electron energy spread [15], although it will be seen that self-amplified spontaneous emission (SASE) [1] is also present.

The radiation may be split into three different regions of $\bar{z}_{1}$ :

(i) The slippage region $0<\bar{z}_{1}<\bar{z}$.

(ii) The steady-state, or cw region $\bar{z}<\bar{z}_{1}<\bar{l}_{e}$.

(iii) The vacuum region $\bar{z}_{1}>\bar{l}_{e}$.

In the slippage region $\left(0<\bar{z}_{1}<5\right)$ CSE from the trailing edge of the electron pulse is amplified as it propagates forward through the electron pulse. Here the rectangular edge of the electron pulse at $\bar{z}=0$ generates intense CSE that may act as a strong seed field which when amplified via SACSE, as here, dominates any SASE process.

The steady-state region $\left(5<\bar{z}_{1}<6\right)$ has effectively only evolved from shot noise as CSE pulse effects, arising from the sharp current gradient at $\bar{z}_{1}=0$, have not yet propagated into this region. It is worth noting that the CSE mechanism does induce an oscillatory amplitude in $|A|^{2} \approx 16 \rho^{2}$ [13]. This oscillation, uniform throughout the steady-state region, has the same period as that of the undulator which, in the scaled variables used here, corresponds to a period in $\bar{z}$ of $4 \pi \rho$. The oscillation arises from the successive constructive then destructive interference of the field in the steady-state region over the undulator period. Because there is no current gradient the constructive/destructive interference cycle is almost perfect and there is no net amplification. Therefore, the only field available for amplification is that arising from shot noise and leads to only SASE in the steady-state region.

In the vacuum region $\bar{z}_{1} \geq 6$ there are no electrons and the radiation propagates in vacuum without further interaction except for the small region around $\bar{z}_{1} \approx 6$, where electrons propagate due to the spread in $p$. The effect of this spread smears out the initially sharp current gradient at $\bar{z}_{1}=6$ as the electron pulse propagates in $\bar{z}$. This smearing of the electron pulse edge consequently reduces the CSE emitted into vacuum as the electron pulse propagates through the interaction region. This effect can be seen from the higher CSE intensities emitted into vacuum at the earlier stages (larger values of $\bar{z}_{1}$ ) of the interaction.

The main features of the PSD are the radiation emission at the fundamental resonant frequency $(f=1)$ with no resonant interaction at the harmonics as expected for a helical undulator, and the significant contributions at low frequencies, $f \lesssim 0.25$. This low frequency emission has also been observed in simulations of the planar undulator [9] and where resonant emission at harmonics is observed. The radiation pulse intensity can be observed in the absence of lower frequency fields by Fourier filtering the fields $A_{x}$ and $A_{y}$ before calculating $|A|^{2}=A_{x}^{2}+A_{y}^{2}$. The SDDS tool kit function SDDSFDFILTER was used to
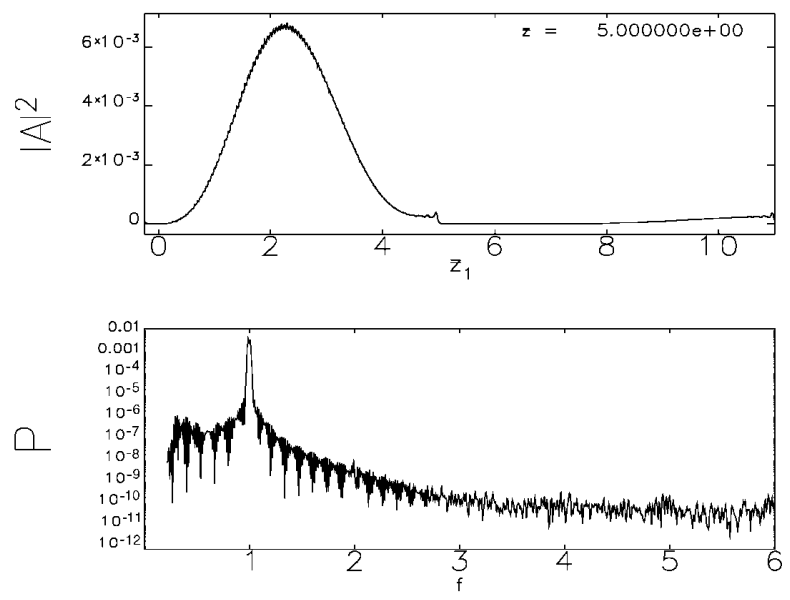

FIG. 5. Top panel: The filtered, scaled intensity, $|A|^{2}$, as a function of scaled pulse position $\bar{z}_{1}$ for a scaled distance through the interaction region of $\bar{z}=5$ and a rectangular profile electron pulse duration of $\bar{l}_{e}=6$ between $0<\bar{z}_{1}<6$. The slippage region is $0<\bar{z}_{1}<5$; the steady-state region is $5<$ $\bar{z}_{1}<6$; the vacuum region is $\bar{z}_{1}>6$. Bottom panel: The filtered scaled PSD, $P$, of the radiation as a function of the scaled frequency $f$.

perform a high pass filter operation on the data $A_{x}$ and $A_{y}$. A linear transition from zero pass at $f=0.2$ to full pass at $f=0.3$ was used. The filtered scaled pulse intensity and PSD are shown in Fig. 5. Comparison of the unfiltered Fig. 4 and the filtered Fig. 5 shows the modulation of the resonant field intensity envelope in Fig. 4 is caused by the low frequency contribution to the field. While we consider these effects worthy of further investigation we will not do so here.

Figure 6 plots both the filtered scaled average radiation intensity $\left\langle|A|^{2}\right\rangle$ and the modulus of the bunching

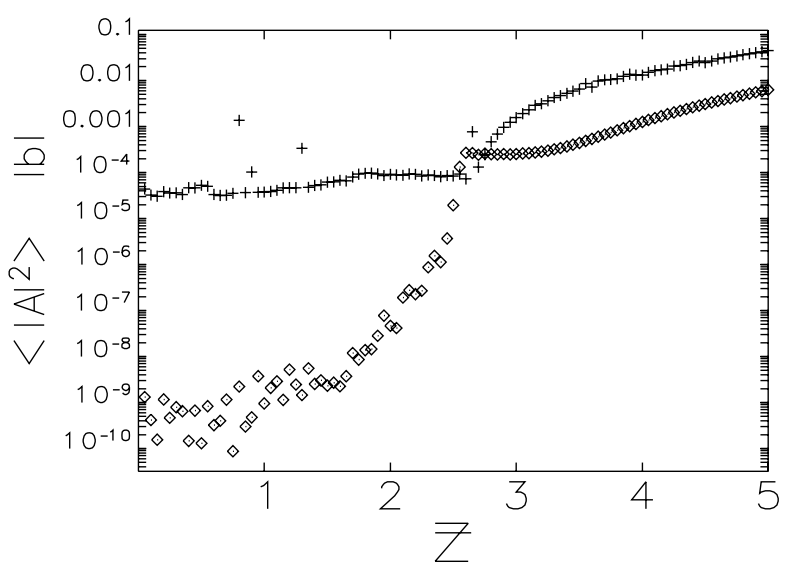

FIG. 6. The filtered scaled average radiation intensity $\left\langle|A|^{2}\right\rangle$ $(\diamond)$ and modulus of the bunching parameter $|b|(+)$ over the single period interval $2.5<\bar{z}_{1}<2.6$ of a rectangular profile electron pulse of $\bar{l}_{e}=6$. For $\bar{z}<2.5$ the interval is in the steady-state region, and for $\bar{z}>2.5$ the slippage region. 
parameter $|b|$ calculated over the single ponderomotive period in the interval $2.5<\bar{z}_{1}<2.6$ as a function of $\bar{z}$. The filtered data of Fig. 5 were used, so that scaled frequencies $f<0.2$ are not present. From the above definitions of the three regions of evolution it is seen that this region in $\bar{z}_{1}$ of the electron pulse will evolve as the steady state (SASE) for $0<\bar{z}<2.5$ and as a slippage region (SASE and SACSE) for $\bar{z}>2.5$. The transition between these two regimes is clearly visible in Fig. 6 from both the radiation and electron properties at $\bar{z} \approx 2.5$.

For the 60 radiation period length rectangular electron pulse of $1 \mathrm{nC}$ used here, the number of electrons per period $\bar{N}_{\lambda} \approx 1.04 \times 10^{8}$ which, from the previous section, gives an expectation of the mean bunching due to shot noise of $\bar{b}=\sqrt{\pi / 4 \bar{N}_{\lambda}} \approx 8.7 \times 10^{-5}$. This is seen to be in reasonable agreement with the bunching in the steady-state regime of Fig. 6. Notice that in the steady state up to $\bar{z} \approx 1.5$ bunching does not significantly evolve about this mean value and that the radiation intensity has significant fluctuations. This is consistent with the electrons emitting spontaneous radiation in the absence of any FEL instability. The lack of the FEL instability in this steady-state region up to $\bar{z} \approx 1.5$ can be attributed to lethargy in the presence of the relatively large electron energy spread parameter $\sigma_{p}=0.5$. In the scaled variables used here the radiation intensity due to shot noise can be estimated as [14]

$$
\left\langle\left|A_{0}\right|^{2}\right\rangle \approx \frac{6 \sqrt{\pi} \rho}{\bar{N}_{\lambda} \sqrt{\ln \left(\bar{N}_{\lambda} / \rho\right)}}
$$

which for the parameters of Fig. 6 gives $\left\langle\left|A_{0}\right|^{2}\right\rangle \approx 1.7 \times$ $10^{-10}$, in reasonable agreement with the numerical simulation values at the beginning of the interaction $\bar{z} \gtrsim 0$.

For $\bar{z} \gtrsim 1.5$ gain of the radiation field is apparent in the transition to $\bar{z} \approx 2.5$ and it can be seen that both electron bunching and radiation appear less noisy. This is thought to be, in part, due to the onset of the FEL instability.

For $\bar{z}>2.5$ the electrons experience CSE radiation from the slippage region and enter the SACSE regime of evolution $[13,15]$. Following a transition region $2.5<$ $\bar{z}<3.5$ both the electron bunching and the radiation intensity are seen to interact collectively with both experiencing nonlinear gain.

A similar FEMFEL run to the above has also been carried out for an electron pulse with a Gaussian current profile of scaled width in $\bar{z}_{1}$ of $\sigma_{z 1}=3$, of total length $\bar{l}_{e}=6 \sigma_{z 1}=18$ centered at $\bar{z}_{1}=\bar{z}_{1 c}=9$. The Gaussian energy spread and $\rho$ parameter are as for the rectangular case above.

It is easily shown that the wave equation is that of (23) but with the normalization factor $\bar{l}_{e}$ on the right-hand side replaced by $\sqrt{2 \pi} \sigma_{z 1}$, and the equivalent expression for $\bar{N}_{j}$ (24) is

$$
\begin{aligned}
\bar{N}_{j}= & \frac{\bar{N}}{\sqrt{2 \pi} \sigma_{z 1}} \exp \left(-\frac{\left(\bar{z}_{1 j}-\bar{z}_{1 c}\right)^{2}}{2 \sigma_{z 1}^{2}}\right) \frac{1}{\sqrt{2 \pi} \sigma_{p}} \\
& \times \exp \left(-\frac{p_{g j}^{2}}{2 \sigma_{p}^{2}}\right) \Delta p \Delta \bar{z}_{1} .
\end{aligned}
$$

The same type of grid in $\left(\bar{z}_{1}, p\right)$ as was used for the rectangular pulse was loaded with macroparticles using this Gaussian profile relation for $\bar{N}_{j}$.

The scaled radiation intensity and PSD from the Gaussian pulse is shown in Fig. 7 for a scaled interaction distance through the interaction region of $\bar{z}=2$. In contrast to the previous rectangular electron pulse profile, there is no steady-state region of evolution. The region that nearest approaches steady state is that around the peak of the electron current at $\bar{z}_{1} \approx 9$ where the current gradient is smallest. As with the rectangular electron pulse profile, the PSD indicates the presence of significant lower frequency emission for $f<1$, in particular, for $f \geq 0$. The same Fourier domain filtering as for the rectangular profile was applied and the result plotted in Fig. 8. It is seen that the presence of lower frequencies appear to have a more profound effect on $|A|^{2}$ for the Gaussian pulse profile than for a rectangular. The regions $0<\bar{z}_{1}<2$ and $18<\bar{z}_{1}<20$ are the CSE resulting from a truncation of the Gaussian electron pulse. This truncation causes discontinuities in the electron pulse current that drive CSE. The region $2<\bar{z}_{1}<18$ has evolved in the presence of both CSE and shot noise. It can be seen that the noisy nature of the emission indicates that the spontaneous shot-noise radiation dominates that of CSE. This is consistent with previous works (e.g., $[10,14])$ which require $|E(b)|^{2}>1 / \bar{N}$ for CSE to dominate shot noise. For the Gaussian case it is easy to show that this requires $\sigma_{z 1}<$
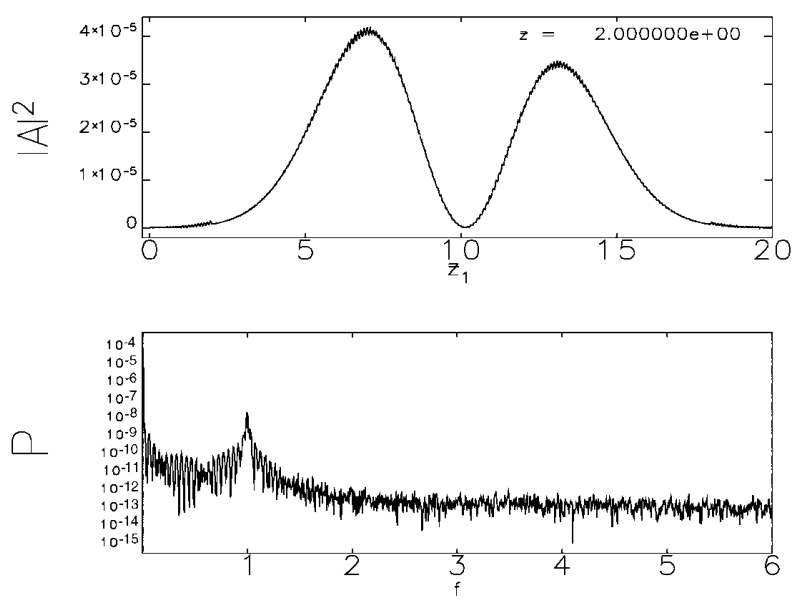

FIG. 7. Top panel: The scaled intensity, $|A|^{2}$, as a function of scaled pulse position $\bar{z}_{1}$ for a scaled distance through the interaction region of $\bar{z}=2$ and a Gaussian profile electron pulse of total duration $\bar{l}_{e}=18$ and $\sigma_{e}=3$. Bottom panel: The scaled PSD, $P$, of the radiation as a function of the scaled frequency $f$. 

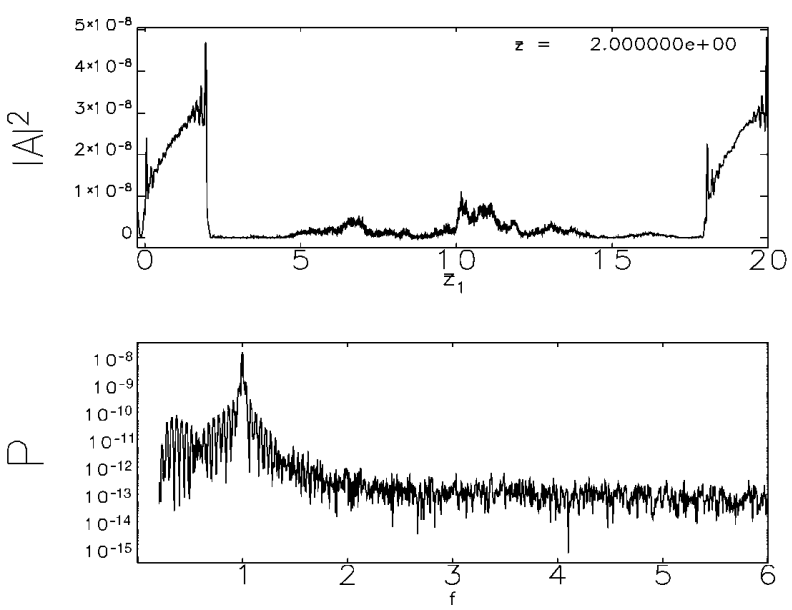

FIG. 8. Top panel: The filtered scaled intensity, $|A|^{2}$, as a function of scaled pulse position $\bar{z}_{1}$ for a scaled distance through the interaction region of $\bar{z}=2$ and a Gaussian profile electron pulse of total duration $\bar{l}_{e}=18$ and $\sigma_{e}=3$. Bottom panel: The filtered scaled PSD, $P$, of the radiation as a function of the scaled frequency $f$.

$2 \rho \sqrt{\ln (\bar{N})}$ for the resonant frequency. For the parameters used here $\sigma_{z 1}=3$ and $2 \rho \sqrt{\ln (\bar{N})} \approx 7.6 \times 10^{-2}$ so that the shot noise should dominate CSE as observed.

The peak number of electrons per period is at $\bar{z}_{1}=9$ with $\bar{N}_{\lambda}=\bar{N} / \sqrt{2 \pi} \sigma_{z 1} \approx 8.3 \times 10^{8}$ which gives an expectation for the bunching due to shot noise of $\bar{b}=$ $\sqrt{\pi / 4 \bar{N}_{\lambda}} \approx 3.1 \times 10^{-5}$. It can be seen from Fig. 9 that the bunching as calculated over the period $8.95<\bar{z}_{1}<$ 9.05 is very slightly larger than this, the difference being attributed to the statistical fluctuations. The scaled average radiation intensity $\left\langle|A|^{2}\right\rangle$ over the same interval is also plotted. The scaled radiation intensity due to shot noise can be estimated, as for the rectangular pulse profile case, from Eq. (28) to obtain $\left\langle\left|A_{0}\right|^{2}\right\rangle \approx 2.0 \times 10^{-11}$. The

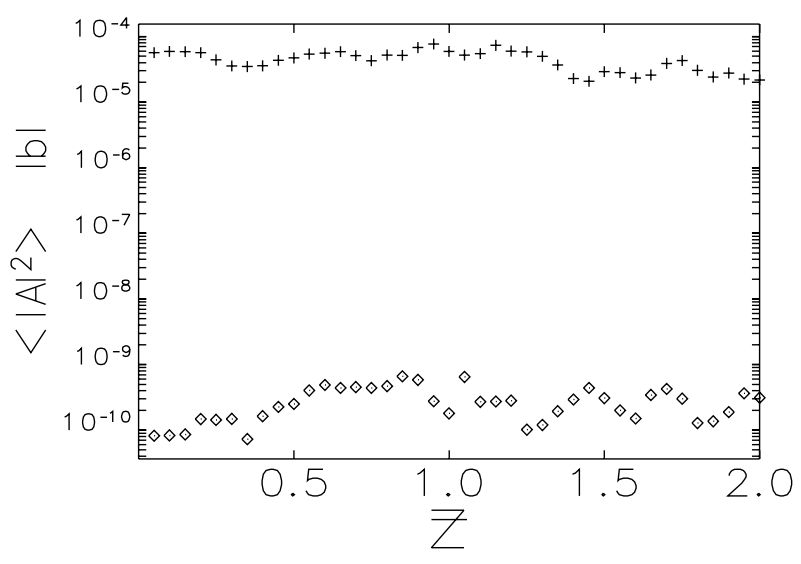

FIG. 9. The filtered scaled average radiation intensity $\left\langle|A|^{2}\right\rangle$ $(\diamond)$ and modulus of the bunching parameter $|b|(+)$ over the single period interval $8.95<\bar{z}_{1}<9.05$ of a Gaussian profile electron pulse of $\bar{l}_{e}=18$ and $\sigma_{e}=3$. value obtained from numerical calculation is a factor $\approx 4$ times this value for $\bar{z} \gtrsim 0$. Again we attribute this difference to statistical fluctuations.

\section{CONCLUSIONS}

The derivation of the shot-noise model presented here is perhaps more physically intuitive, and therefore appealing, than those used in current FEL simulation codes: The macroparticle properties of arrival time and charge are derived directly from the intrinsic Poisson statistical properties of the individual electron arrival times at the beginning of the interaction region. The model is therefore independent of any external factors such as a resonant radiation period. A simple numerical example including energy spread demonstrated that the model describes well the averaged bunching statistics of a real electron distribution.

The shot-noise algorithm was further tested in the 1D code FEMFEL currently under development to describe simultaneously the effects of shot noise, CSE, and electron beam energy spread in a single pass FEL. Preliminary results from this code demonstrate good qualitative and quantitative agreement with that expected from analysis and have shown that both SASE and SACSE evolution may coexist within the same rectangular profile electron pulse. Work is required to further test the model for correct statistical behavior of fluctuations and higher order correlations [14].

Further validation of the model to higher dimensions of phase space will be required in FEL codes such as GENESIS 1.3 [3] and GINGER [2]. Some modification of the model may be required when applied to such timeaveraged codes, especially when harmonic radiation is to be modeled, where quite complex macroparticle loading schemes are necessary [5]. It is not apparent however, that unaveraged radiation field models such as FEMFEL should require such complex loading schemes as the full frequency content of the radiation is contained within the single scaled complex radiation field $A\left(\bar{z}, \bar{z}_{1}\right)$. In contrast to averaged models, where the radiation field is usually described by a summation over harmonic modes with each mode requiring the solution of a PDE, by using the FEM there is only one complex PDE describing all harmonic radiation evolution. The harmonic content of this field is limited only by the size of the finite elements used to discretize the field via the Nyquist theorem.

Another area that would benefit from further investigation is the presence in the model of significant field amplitudes at frequencies well below the fundamental. The existence of these low frequency fields is not an artifact of the numerical model as this model agrees very well with the results of analytical analysis $[9,13]$.

Finally, it should be noted that it may be possible to reduce the number of macroparticles required in high dimensional simulations by either carrying out a 
quasirandom sampling of phase space [20] or by discretizing phase space so that elemental volumes are not equal. One method, for example, would be to discretize phase space so that each volume element $\Delta V_{\alpha}$ of Eq. (22) contains approximately equal numbers of electrons $\bar{N}_{j}$. In some circumstances this method may also assist in improving the statistics by reducing the charge contrast between macroparticles where this is an important factor.

[1] R. Bonifacio, C. Pelligrini, and L. M. Narducci, Opt. Commun. 50, 373 (1984).

[2] W. M. Fawley, Lawrence Berkeley National Laboratory Report No. LBNL-49625, 2002.

[3] S. Reiche, Nucl. Instrum. Methods Phys. Res., Sect. A 429, 243 (1999).

[4] C. Penman and B.W. J. McNeil, Opt. Commun. 90, 82 (1992).

[5] William M. Fawley, Phys. Rev. ST Accel. Beams 5, 070701 (2002).

[6] The TESLA test facility FEL team, TESLA-FEL Report No. 2002-01, 2002, DESY, Hamburg, Germany.

[7] J. Arthur et al., Stanford Linear Accelerator Center Report No. SLAC-R-593 UC-414, 2002.

[8] M.W. Poole and B.W. J. McNeil, Nucl. Instrum. Methods Phys. Res., Sect. A 507, 489 (2003); (see also www.4GLS.ac.uk).
[9] B.W. J. McNeil and G. R. M. Robb, Phys. Rev. E 65, 046503 (2002); 66, 059902(E) (2002).

[10] B.W. J. McNeil and G. R. M. Robb, J. Phys. D 30, 567 (1997); 31, 371(E) (1998).

[11] W. B. Davenport, Probability and Random Processes (McGraw-Hill, London, 1970).

[12] John E. Freund, Mathematical Statistics (Prentice-Hall, London, 1972), 2nd ed.

[13] B.W. J. McNeil, G. R. M. Robb, and D. A. Jaroszynski, Opt. Commun. 165, 65 (1999).

[14] E. L. Saldin, E. A. Schneidmiller, and M.V. Yurkov, TESLA-FEL Report No. 97-02, 1997, DESY, Hamburg, Germany.

[15] B.W. J. McNeil, G. R. M. Robb, and D. A. Jaroszynski, Nucl. Instrum. Methods Phys. Res., Sect. A 445, 72 (2000).

[16] M. Borland, in Proceedings of the 1995 Particle Accelerator Conference, Dallas, TX (IEEE, Piscataway, NJ, 1996), p. 2184.

[17] M. Borland, OAG Software Documentation, http:// www.aps.anl.gov/asd/oag/oagSoftware.html

[18] C. Johnson, Numerical Solution of Partial Differential Equations by the Finite Element Method (Cambridge University Press, Cambridge, 1995).

[19] E. A. Huebner, E. A. Thornton, and T. G. Byrom, The Finite Element Method for Engineers (Wiley, New York, 1995).

[20] S. Reiche, Ph.D. thesis, DESY, Hamburg, Germany, 1999. 